\title{
FUNCIONES DE BIOMASA PARA SEIS ESPECIES ARBUSTIVAS DE LA ZONA ARIDA CHILENA
}

\author{
José A. Prado D. (*) \\ Pedro Infante L. $\left.{ }^{* *}\right)$ \\ Manuel Arriagada B. $\left({ }^{* *}\right)$ \\ Sergio Aguirre. $\left({ }^{*}\right)$
}

\begin{abstract}
RESUMEN
Como parte de un estudio que pretende cuantificar la disponibilidad de biomasa en un sector de la zona árida chilena, se desarrollan funciones para estimar la biomasa de seis especies consideradas imporțantes, que son: Acacia caven, Cassia coquimbensis, Colliguaya odorifera, Cordia decandra, Flourenzia thurifera y Lithraea caustica. Se seleccionan modelos lineales ya que entregan mejores estimaciones que los modelos logarítmicos. Considerando la gran variabilidad que presentan las especies en cuanto a su edad, forma y grado de alteración por la acción del hombre y los animales, las funciones entregan una buena estimación de la biomasa, la que, en los arbustos mayores. se concentra en las ramillas $(<3 \mathrm{~cm})$ y hojas.
\end{abstract}

\begin{abstract}
Biomass equations to estimate weight of six shruby species were developed as part of a project that intends to quantify the availability of fuelwood in an area of the Chilean arid zone. Linear models were selected since they gave better estimates than logarithmic functions.

Good biomass estimates were obtained in spite of the great variability that each species presents due to age variations and alterations caused by man and animals. Results indicate that in tall shrubs, biomass is mainly concentrated in small branches (Diameter $<3 \mathrm{~cm}$ ) and leaves.
\end{abstract}

(*) Ingenieros Forestales. División Silvicultura, Instituto Forestal. Huérfanos 554. Piso 4. Santiago - Chile

(**) Licenciados en Ingenicría Forestal, División Silvicultura, Instituto Forestal. Huérfanos 554 - Piso 4. Santiago Chile. 


\section{INTRODUCCION}

Las formaciones vegetales naturales de la zona árida chilena se caracterizan por una baja densidad, un escaso desarrollo de la mayoría de las especies y normalmente, por un avanzado estado de degradación producto de una sobre-utilización a que han sido tradicionalmente sometidas. A pesar de esta condición, estas formaciones vegetales aún constituyen un recurso fundamental para la subsistencia de muchas comunidades rurales, ya que son la principal fuente de energia, forraje, madera para infraestructura y otros bienes.

Este hecho hace que exista una intensa y constante presión sobre estas formaciones, lo cual ha producido, en muchos casos, una fuerte degradación e incluso la eliminación de parte 0 del total de la vegetación, especialmente cerca de las áreas más densamente pobladas. A esta presión se debe agregar el desconocimiento de aspectos relacionados con la estructura, regeneración y desarrollo de las distintas formaciones vegetales, la cual impide desarrollar esquemas de manejo racional, que permitan la utilización del recurso sin poner en peligro su existencia.

La descripción y cuantificación de la vegetación son pasos fundamentales para iniciar estudios que, con una base ecológica, conduzcan a la determinación de pautas de manejo que permitan obtener de este recurso bienes tales como leña, forraje, postes, productos químicos u otros, sin que se produzca un daño, muchas veces irreparable, en la vegetación o en el suelo que la sustenta.

Se han realizado algunos trabajos que describen la vegetación de ciertos sectores del área de estudio. ETIENNE et al.(1982, 1982a) realizaron una cartografía de la vegetación sobre varios transectos representativos de distintas formaciones vegetales. IREN (1979) en su estudio de las Comunidades Agrícolas de la IV Región, también incluye una descripción de la vegetación.

Este trabajo forma parte de uno de los primeros intentos por cuantificar la existencia de biomasa en una zona de la IV Región ocupada por Comunidades Agrícolas, con el objeto de analizar la presión directa que la población ejerce sobre la vegetación, a través de su empleo como combustible.

Una herramienta fundamental para esta cuantificación son las funciones que permitan estimar la biomasa total o de los distintos componentes de las especies que se encuentran en mayor abundancia dentro del área estudiada, a partir de variables de fácil obtención. Estas funciones también constituyen la base para el estudio de la productividad biológica de las formaciones vegetales, lo cual tiene importantes implicaciones en el desarrollo de cualquier sistema de manejo de la vegetación (RIGGAN and DUNN, 1982).

\section{MATERIAL Y METODO}

\section{El Area de Estudio}

Tomando como base el estudio realizado por ETIENNE et al. (1982) se eligieron dos de los transectos definidos, de los cuales existe una completa descripción de la vegetación. Los transectos, denominados Cogotí - Guatulame y Guatulame - Embalse La Paloma, están ubicados en la provincia de Limarí, entre los $30^{\circ} 40^{\circ}$ y los $31^{\circ} 08^{\prime}$ L.S., siguiendo el curso del río Guatulame.

El área de estudio tiene una superficie total de aproximadamente $16.000 \mathrm{ha}$, de las cuales alrededor de un $50 \%$ presenta vegetación de interés para los fines del estudio.

\section{Especies Seleccionadas}

Agrupando los tipos definidos por ETIENNE et al. (1982) se obtienen 20 tipos vegetacio- 
nales diferentes, dados por distintas combinaciones de especies y grados de cobertura. Las especies que por presencia y área ocupada se consideraron de mayor interés son las siguientes:

\begin{abstract}
Acacia caven
Cassia coquimbensis

Colliguaya odorifera

Cordia decandra

Flourenzia thurifera

Lithraea caustica
\end{abstract}

(Mimosaseae)

(Caesalpinaceae)

(Euphorbiaceae)

(Boraginaceae)

(Compositae)

(Anacardiaceae)
"Espino"
"Alcaparra"
"Colliguay"
"Carbonillo"
"Maravilla"
"Litre"

De las especies seleccionadas, espino, carbonillo y litre son arbustos grandes y a veces pequeños árboles; las restantes son arbustos que en el área de estudio alcanzan escaso desarrollo.

\section{Muestreo}

En base a la descripción realizada por ETIENNE et al. (1982) y a un recorrido general por el área de estudio, se seleccionaron sectores representativos para realizar el muestreo de 40 individuos de cada una de las especies antes mencionadas.

Con el fin de obtener una buena distribución de la muestra, se realizó una medición previa de por lo menos 150 individuos por especie en 3 parcelas de tamaño variable, dependiendo de la densidad de la vegetación. Se midió el diámetro mayor de la copa de todos los individuos, los que, de acuerdo a esta variable, fueron agrupados en cinco clases diamétricas. La selección de los 40 individuos por especie se hizo de la siguiente manera: 25 fueron seleccionados en forma dirigida, en igual número por clase y los 15 restantes al azar, pero con probabilidad proporcional a la frecuencia por el diámetro de copa al cuadrado y por la altura ( $\mathrm{F}$ ${ }^{*} \mathrm{DMAC}^{2}{ }^{*} \mathrm{H}$ ), con lo que se pretende dar una mayor probabilidad de asignación a las clases mayores y a aquellas que representen la mayor cantidad de individuos.

\section{Obtención de peso verde y peso seco}

Una vez seleccionados los individuos a muestrear se midieron las variables que se indican en la Tabla 1 según especie:

TABLA 1

VARIABLES INDEPENDIENTES MEDIDAS SEGUN ESPECIE

\begin{tabular}{|c|c|c|c|c|c|c|c|}
\hline \multirow[b]{2}{*}{ Especies } & \multicolumn{7}{|c|}{ VARIABLES } \\
\hline & $\begin{array}{c}\mathrm{DB} \\
(\mathrm{cm})\end{array}$ & $\begin{array}{l}\text { HT } \\
(\mathrm{m})\end{array}$ & $\begin{array}{c}\mathrm{AMF} \\
(\mathrm{m})\end{array}$ & $\begin{array}{c}\text { NR } \\
-\end{array}$ & $\begin{array}{c}\text { LXR } \\
(\mathrm{m})\end{array}$ & $\begin{array}{c}\text { DMEC } \\
(\mathrm{m})\end{array}$ & $\begin{array}{c}\text { DMAC } \\
\text { (m) }\end{array}$ \\
\hline Carbonillo & $\mathrm{x}$ & $\mathrm{x}$ & $x$ & $x$ & $x$ & $x$ & $\mathrm{x}$ \\
\hline Espino & $\mathrm{x}$ & $x$ & $x$ & $\mathrm{x}$ & $x$ & $\mathrm{x}$ & $x$ \\
\hline Litre & $\mathrm{x}$ & $x$ & $\mathrm{x}$ & $\mathrm{x}$ & $x$ & $\mathrm{x}$ & $\mathrm{x}$ \\
\hline Alcaparra & & $x$ & $x$ & & & $x$ & $\mathrm{x}$ \\
\hline Colliguay & & $x$ & $x$ & & & $\mathrm{x}$ & $\mathrm{x}$ \\
\hline Maravilla & & $\mathrm{x}$ & $\mathrm{x}$ & & & $\mathrm{x}$ & $\mathrm{x}$ \\
\hline
\end{tabular}

: Diámetro medio en la base, medido a unos $5 \mathrm{~cm}$ del suelo a las ramas con diámetros iguales o mayores a $3 \mathrm{~cm}$. 
HT : Altura total, medida hasta el punto más alto del arbusto.

HMF : Altura de máximo follaje, medida hasta el punto en que la copa aún se presenta relativamente densa.

NR : Número de ramas con diámetros de $3 \mathrm{~cm}$ o más, contadas en la base del arbusto.

LXR : Longitud media de las ramas, tomada como la distancia más corta entre la base y el extremo de una rama.

DMEC : Diámetro menor de copa

DMAC : Diámetro mayor de copa

Los modelos empleados fueron de 2 tipos:

a) Alométricos, con las siguientes variaciones:

$$
\begin{aligned}
& Y=b_{0} x^{b_{1}} \in . \\
& Y=b_{0}\left(x_{1}^{2} x_{2} b^{b_{1}} \in .\right. \\
& Y=b_{0} x_{1} b_{1} x_{2} b_{2} \in .
\end{aligned}
$$

Todos estos modelos fueron ajustados mediante transformación logarítmica, asumiendo que el error $(E)$ es multiplicativo (BASKERVILLE, 1972) y por lo tanto corregidos de acuerdo al procedimiento dado por WIANT and HARNER (1979) para compensar el sesgo producido por la transformación.

b) Lineales, de la forma:

$$
Y=b_{0}+b_{1} x_{1}+b_{2} x_{2}+\cdots+b_{n} x_{n}
$$

La selección de las variables a ser incluidas en cada función se hizo empleando el método de regresión paso a paso (step - wise).

Para la selección de los mejores modelos se comparan los coeficientes de determinación $\left(\mathrm{R}^{2}\right)$, el Error Cuadrático Medio (ECM) y el porcentaje de observaciones con errores menores de 10,15 y $20 \%$. Además se considera la presencia de sesgos. Entre todos estos indicadores se da mayor importancia al ECM, ya que incluye los errores aleatorios y sistemáticos, representando adecuadamente la exactitud del estimador.

Después de obtener las variables independientes se procedió a la obtención del peso verde. Cada uno de los arbustos seleccionados fue cortado e inmediatamente pesado.

En el caso de los arbustos de mayor tamaño (Litre, Espino, Carbonillo) la biomasa fue dividida en dos componentes:

Ramas : Material leñoso con un diámetro mínimo de $3 \mathrm{~cm}$.

Ramillas : Material leñoso de menos de $3 \mathrm{~cm}$ de diámetro, que incluye hojas y frutos.

Para encontrar una relación peso verde/peso seco que permita estimar el peso seco, se tomaron 12 muestras de cada componente, las que fueron pesadas en terreno con una precisión de $0,1 \mathrm{~g}$.

Los arbustos más pequeños (Colliguay, Maravilla y Alcaparra) fueron cortados y pesados, obteniéndose un peso verde total. También se tomaron las 12 muestras para determinar la relación peso verde/peso seco. 


\section{J.A. PRADO D., P. INFANTE L., M. ARRIAGADA B. y S. AGUIRRE A.}

Las muestras colectadas para conseguir relaciones peso verde/peso seco se secaron en laboratorio a $100^{\circ} \mathrm{C}$, hasta alcanzar peso constante.

\section{Análisis}

Para expresar las relaciones entre el peso y las dimensiones de los arbustos estudiados, se probaron varios modelos, empleando las variables independientes tal como fueron medidas. combinadas o transformadas ( $\mathrm{Ln}$ ).

\section{RESULTADOS Y DISCUSION}

\section{Funciones de biomasa}

De acuerdo con los criterios enunciados en el punto anterior y también considerando el número y tipo de variables involucradas en cada estimador, se seleccionaron las funciones que se entregan en los cuadros siguientes. Con el fin de obtener funciones de fácil aplicación, se eliminaron aquellas que incluían variables de difícil medición, por ejemplo la longitud promedio de las ramas (LXR).

TABLA 2

FUNCIONES PARA ESTIMAR BIOMASA TOTAL Y DE COMPONENTES DE TRES ESPECIES ARBUSTIVAS MAYORES. PESO VERDE (Kg).

\begin{tabular}{|c|c|c|c|c|c|}
\hline \multirow{2}{*}{$\begin{array}{l}\text { Especie } \\
\text { Carbonillo }\end{array}$} & \multicolumn{2}{|c|}{ Componente } & Función & $\mathbf{R}^{2}$ & ECM \\
\hline & Total & PTOT & $\begin{aligned}= & -17,8305+0,1287(\mathrm{AA})+22,913(\mathrm{DMAC}) \\
& -22,471(\mathrm{HMF})-2,249 \text { (NR) }\end{aligned}$ & 0,92 & 20,6 \\
\hline & Ramas & PRAM & $\begin{aligned}= & -9.4454+0.05326(\mathrm{AA})-0.01549(\mathrm{CC}) \\
& +2.1557(\mathrm{DB})\end{aligned}$ & 0,89 & 34.2 \\
\hline & Ramillas & PRAMI & $\begin{aligned}= & 7,3483+0,939\left(\mathrm{DMAC}^{2 *} \mathrm{HT}\right)-19,511 \text { (HMF) } \\
& +7,6739 \text { (DMAC) }\end{aligned}$ & 0.90 & 25.5 \\
\hline \multirow[t]{3}{*}{ Espino } & Total & PTOT & $=-17,0107+0,05463(\mathrm{AA})+12,82(\mathrm{DMEC})$ & 0,96 & 18,7 \\
\hline & Ramas & PRAM & $=-0,8666+0,05097(\mathrm{AA})$ & 0,97 & 23,3 \\
\hline & Ramillas & PRAMI & $\begin{aligned}= & -10,2917+2,1555(\mathrm{DMAC})+0,03018(\mathrm{DD}) \\
& +6,9923(\mathrm{DMEC})\end{aligned}$ & 0,92 & 19.9 \\
\hline \multirow[t]{3}{*}{ Litre } & Total & PTOT & $=0,1634+1,675\left(\mathrm{DMEC}^{2} * \mathrm{HT}\right)$ & 0,96 & 18,6 \\
\hline & Ramas & PRAM & $\begin{aligned}= & 14,911-0,0037 \text { (AA) }-2,444(\mathrm{NR}) \\
& +8,3126(\mathrm{DMEC})+0,0592(\mathrm{BB})\end{aligned}$ & 0,96 & 19,6 \\
\hline & Ramillas & PRAMI & $=-46,6962+26,692(\mathrm{DMEC})+0,0101(\mathrm{DD})$ & 0,94 & 22,7 \\
\hline
\end{tabular}

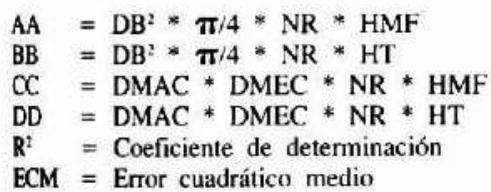

DB = Diámetro basal $(\mathrm{cm})$

$\mathrm{NR}=\mathrm{N}^{\circ}$ de ramas mayores de $3 \mathrm{~cm}$ de 0

$\mathrm{HMF}=$ Altura de máximo follaje $(\mathrm{m})$

HT = Altura total $(\mathrm{m})$

DMAC $=$ Diámetro mayor de copa (m)

DMEC $=$ Diámetro menor de copa $(\mathrm{m})$ 
TABLA 3

\section{FUNCIONES PARA ESTIMAR BIOMASA TOTAL DE TRES ESPECIES ARBUSTIVAS MENORES. PESO VERDE (Kg)}

\begin{tabular}{|l|c|rr|}
\hline Especie & Función & $\mathbf{R}^{2}$ & ECM \\
\hline Alcaparra & $\mathrm{PT}=0.3712+1.8087\left(\mathrm{DMEC}{ }^{2} * \mathrm{HT}\right)-0.6463(\mathrm{DMEC})$ & & \\
& $\mathrm{HT}=5.09754+1.2332(\mathrm{DMAC} * \mathrm{DMEC} * \mathrm{HT})-4.8808(\mathrm{HMF})$ & 0.89 & 39,8 \\
Colliguay & $\mathrm{PT}=0.94$ & 20.9 \\
Maravilla & $\mathrm{PT}=0.5811+0.8364(\mathrm{DMAC} * \mathrm{HT})+1.5429(\mathrm{DMAC})$ & $\mathbf{0 . 8 9}$ & 23,1 \\
\hline
\end{tabular}

$\mathrm{PT}=$ Peso total $(\mathrm{Kg})$
$\mathrm{DMAC}=$ Diámetro mayor de copa $(\mathrm{m})$
$\mathrm{DMEC}=$ Diámetro menor de copa $(\mathrm{m})$
$\mathrm{HMF}=$ Altura de máximo follaje $(\mathrm{m})$
$\mathrm{R}^{2}=$ Coeficiente de determinación
$\mathrm{ECM}=$ Error cuadrático medio $(\%)$

Las funciones seleccionadas son del tipo $Y=b_{0}+b_{1} x_{1}+b_{2} x_{2}+\ldots+b_{n} x_{n}$. Las funciones logaritmicas en general entregan errores más altos, aún después de la corrección por sesgo (BASKERVILLE, 1972). Esto se contrapone a la tendencia generalizada a emplear funciones logarítmicas para la estimación de biomasa en árboles y arbustos (BASKERVILLE, 1972; YANDLE and WIANT, 1981).

Teniendo en cuenta la gran variabilidad que presentan las especies en estudio, en cuanto a su forma, edad de los individuos, estado de alteración debido al efecto del hombre y los animales, los errores de estimación (ECM) pueden considerarse aceptables, especialmente en las funciones para estimar peso total en los arbustos mayores. En relación a los valores de error de estimación resulta difícil hacer comparaciones con otros trabajos similares, ya que en la mayoría de los casos no se entregan antecedentes al respecto (WILLIAMS, 1982).

Los valores de $\mathrm{R}^{2}$ en general son altos, e indican una buena correlación entre las variables seleccionadas y el peso verde total.

En el caso de las especies arbustivas mayores (Tabla 2), debido al empleo de distintos modelos para cada componente, las funciones no son aditivas (KOZAC, 1970), es decir, la suma de las estimaciones de los componentes no es igual a la estimación del peso total. Aun cuando las diferencias producidas son casi despreciables es necesario tener presente este hecho, por lo que se sugiere estimar el peso del componente cuya función tenga más probabilidades de error por diferencia.

KOZAC (1970) sugiere emplear un modelo único para todos los componentes y el peso total, para evitar el problema de la falta de aditividad. En este caso se eligió el empleo de modelos diferentes ya que las diferencias producidas son mínimas y el empleo de un modelo único habría aumentado los errores de estimación.

En el caso de las funciones para estimar el peso total de arbustos menores (Tabla 3), los valores del ECM son algo superiores, especialmente en el caso de Alcaparra. Esta especie, en general, se encuentra muy dañada y presenta una gran variabilidad en su forma.

Con el fin de encontrar funciones más adecuadas para la estimación del peso total de esta especie se probaron funciones ponderadas, empleando el factor I (DMAC ${ }^{*} \mathrm{HT}$ ) (CROW and LAIDLY, 1980), pero no se logró obtener un mejor estimador.

Las funciones determinadas aparentemente son muy complejas; pero dada la facilidad de proceso que hoy día ofrecen los computadores todas resultan simples, ya que están basadas en variables de fácil medición, especialmente en los arbustos bajos. 
J. A. PRADO D., P. INFANTE L., M. ARRIAGADA B. y S. AGUIRRE A.

\section{Rangos de validez en la aplicación de las funciones}

Un aspecto importante a considerar en la aplicación práctica de las funciones de biomasa es su rango de validez, es decir, entre qué valores de las variables independientes pueden estimarse con seguridad, de acuerdo a los errores calculados.

\section{TABLA 4}

\section{RANGO DE APLICACION DE LAS VARIABLES INCORPORADAS EN LAS FUNCIONES}

\begin{tabular}{|c|c|c|c|c|}
\hline \multirow{2}{*}{$\begin{array}{l}\text { Especie } \\
\text { Carbonillo }\end{array}$} & \multirow{2}{*}{$\begin{array}{l}\text { Variable } \\
\text { Diámetro Basal }(\mathrm{cm})\end{array}$} & \multirow{2}{*}{$\begin{array}{c}\text { Promedio } \\
4,5\end{array}$} & \multicolumn{2}{|c|}{ Rango } \\
\hline & & & 3,1 & - 6,9 \\
\hline & Número de ramas & 6,7 & 1,0 & $-15,0$ \\
\hline & Altura total (m) & 2,1 & 1,1 & - 3,6 \\
\hline & Altura máximo follaje $(\mathrm{m})$ & 1,9 & 1,0 & $-3,4$ \\
\hline & Diámetro mayor de copa $(\mathrm{m})$ & 3,9 & 1,9 & $-6,0$ \\
\hline & Diámetro menor de copa (m) & 3,2 & 1,5 & $-5,1$ \\
\hline \multirow[t]{6}{*}{ Espino } & Diámetro Basal $(\mathrm{cm})$ & 6,0 & 3,4 & $-11,6$ \\
\hline & Número de ramas & 4,1 & 1,0 & $-10,0$ \\
\hline & Altura total (m) & 2,5 & 1,6 & $-4,0$ \\
\hline & Altura máximo follaje (m) & 2,2 & 1,3 & $-3,5$ \\
\hline & Diámetro mayor de copa $(\mathrm{m})$ & 3,4 & 1,3 & $-5,7$ \\
\hline & Diámetro menor de copa $(\mathrm{m})$ & 2,8 & 1,2 & $-5,1$ \\
\hline \multirow[t]{6}{*}{ Litre } & Diámetro Basal $(\mathrm{cm})$ & 5,3 & 3,3 & $-11,1$ \\
\hline & Número de ramas & 14,6 & 1,0 & $-52,0$ \\
\hline & Altura total $(\mathrm{m})$ & 3,0 & 1,6 & $-5,2$ \\
\hline & Altura máximo follaje (m) & 2,7 & 1,4 & $-5,2$ \\
\hline & Diámetro mayor de copa (m) & 4,8 & 1,5 & - 9,1 \\
\hline & Diámetro menor de copa (m) & 4,3 & 1,3 & $-7,5$ \\
\hline \multirow[t]{3}{*}{ Alcaparra } & Altura total $(\mathrm{m})$ & 0,89 & 0,31 & $-1,60$ \\
\hline & Altura máximo follaje $(\mathrm{m})$ & 0.81 & 0,28 & $-1,44$ \\
\hline & Diámetro menor de copa $(\mathrm{m})$ & 0,81 & 0,26 & - 1,92 \\
\hline \multirow[t]{4}{*}{ Colliguay } & Altura total $(\mathrm{m})$ & 1,39 & 0,54 & - 2,49 \\
\hline & Altura máximo follaje $(\mathrm{m})$ & 1,19 & 0,50 & $-2,40$ \\
\hline & Diámetro mayor de copa $(\mathrm{m})$ & 2,25 & 0.74 & - 3,89 \\
\hline & Diámetro menor de copa $(\mathrm{m})$ & 1,86 & 0,67 & - 3,38 \\
\hline \multirow[t]{4}{*}{ Maravilla } & Altura total $(\mathrm{m})$ & 1,01 & 0,39 & $-1,70$ \\
\hline & Altura máximo follaje (m) & 0,72 & 0,35 & $-1,15$ \\
\hline & Diámetro mayor de copa $(\mathrm{m})$ & 1.13 & 0,42 & $-1,99$ \\
\hline & Diámetro menor de copa (m) & 0.90 & 0,31 & - 1,80 \\
\hline
\end{tabular}

\section{Obtención de pesos secos}

Las muestras colectadas para obtener las relaciones peso verde/peso seco, se secaron en laboratorio hasta obtener peso constante. Con estos datos se calcularon los factores de conversión que se entregan en la Tabla 5. 
TABLA 5

FACTORES DE CONVERSION PESO SECO/PESO VERDE, SEGUN ESPECIE Y COMPONENTE

\begin{tabular}{lccc}
\hline Especie & Ramas & Ramillas (1) & Hojas \\
\hline Alcaparra & - & 0,66 & 0,46 \\
Colliguay & - & 0,71 & 0,63 \\
Maravilla & - & 0,82 & 0,76 \\
Carbonillo & 0,67 & 0,70 & 0,70 \\
Espino & 0,62 & 0,68 & 0,80 \\
Litre & 0,65 & 0,60 & 0,56 \\
\hline
\end{tabular}

NOTA: (1) Incluyen hojas

En general llama la atención el escaso contenido de humedad de la mayoría de los componentes de las diferentes especies. Sólo las hojas de Alcaparra y Litre presentan contenidos de humedad relativamente altos.

En el área de estudio, las relaciones peso verde/peso seco pueden variar considerablemente de un año a otro y dentro del año. Estas muestras fueron tomadas al final del verano, de un año extremadamente seco. Sin duda que durante la primavera y en un año con precipitación normal, los contenidos de humedad pueden ser muy superiores.

\section{Distribución de la biomasa}

En base a los valores medios de los datos obtenidos en terreno y a las estimaciones hechas con las funciones seleccionadas, se determinó la distribución de la biomasa en los componentes de las especies arbustivas mayores. Esta información se entrega en la Tabla 6.

TABLA 6

\section{DISTRIBUCION DE LA BIOMASA EN LOS COMPONENTES DE LAS ESPECIES ARBUSTIVAS MAYORES ( $\%$ del peso total)}

\begin{tabular}{lllccc}
\hline & & \multicolumn{2}{c}{ Peso Verde } & \multicolumn{2}{c}{ Peso Seco } \\
& & $\mathrm{XR}$ & $\mathrm{XE}$ & $\mathrm{XR}$ & $\mathrm{XE}$ \\
\hline Carbonillo & & & & & \\
& Ramas & 20,26 & 20,20 & 19,56 & 19,50 \\
\multirow{3}{*}{ Espino } & Ramillas & 79,74 & 79,80 & 80,44 & 80,50 \\
& Ramas & 42,02 & 42,03 & 39,79 & 39,80 \\
\multirow{4}{*}{ Litre } & Ramillas & 57,98 & 57,97 & 60,21 & 60,20 \\
& Ramas & 35,29 & 35,29 & 37,14 & 37,14 \\
& Ramillas & 64,71 & 64,71 & 62,86 & 62,86 \\
\hline
\end{tabular}

NOTA: $\mathrm{XR}=$ Valores reales; $\mathrm{XE}=$ Valores estimados 
Del análisis de los datos entregados en la Tabla 6 , se concluye que la mayor parte de la biomasa está contenida en las ramillas, es decir, en el material leñoso de menos de $3 \mathrm{~cm}$ de diámetro, incluyendo hojas $y$, en algunos casos, frutos.

El espino es la especie que mucstra la proporción más alta de material grueso, con un $42 \%$ del peso total verde. En el caso del peso seco. esta proporción disminuye ligeramente. debido al escaso contenido de humedad de las ramillas. Al contrario, el Carbonillo sólo alcanza una proporción cercana al 20\%. El Litre está en una situación intermedia, con un $35 \%$ del peso verde en material grueso, cifra que aumenta ligeramente en el caso del peso seco.

No existen diferencias importantes entre los valores reales y los valores estimados.

\section{CONCLUSIONES}

Considerando la gran variabilidad que presentan las especies estudiadas en cuanto a su edad, forma y grado de alteración. las funciones de biomasa seleccionadas pueden considerarse como buenos estimadores, especialmente las de peso total de los arbustos mayores.

Frente a esta gran variabilidad. sólo es posible disminuir los errores de estimación. aumentando considerablemente el tamaño de las muestras.

La función para estimar el peso total de la Alcaparra es la única que puede arrojar un error bastante alto, pero este hecho pierde importancia dentro del objetivo general del trabajo, ya que esta especie es bastante escasa y de muy poco desarrollo dentro del área de estudio.

Como se mencionó anteriormente, las relaciones peso verde/ peso seco pueden estar sujetas a importantes variaciones de año en año y de acuerdo a la época en que se haga el muestreo. Este hecho hace recomendable tomar algunas muestras al momento de tomar los datos para un inventario de biomasa. para poder obtener una estimación de peso seco más ajustado.

Este mismo hecho puede afectar la bondad de las estimaciones de peso verde, que pueden resultar en una subestimación generalizada, si es que el muestreo se realiza cuando las plantas disponen de un máximo de humedad. Esto último debido a que el muestreo para determinar las funciones fue hecho en pleno período seco y en un año con precipitaciones por debajo de lo normal.

Las especies arbustivas mayores concentran la mayor parte de su biomasa en las ramillas delgadas $(<3 \mathrm{~cm})$ y en las hojas. Desde el punto de vista de la producción de leña y carbón. el Espino resulta la especie más adecuada ya que es la que concentra el mayor porcentaje de su biomasa en las ramas gruesas; lo opuesto ocurre con Carbonillo.

Se debe tener presente que los datos que generen las funciones de Litre y Espino provienen de individuos que alguna vez fueron cortados y retonaron del tocón. Por esto, es altamente probable que los errores de estimación de las funciones aumenten en forma considerable si se aplican en individuos con hábito arbóreo. Asimismo, las condiciones del sitio en donde las especies están creciendo modifican las relaciones entre los parámetros considerados, por lo que las funciones pueden perder precisión si se aplican en otras zonas del pais, con condiciones muy distintas a las del área de estudio.

\section{RECONOCIMIENTOS}

Este trabajo forma parte del proyecto "Aprovechamiento de la Biomasa en Zonas Aridas y Semiáridas", cuya realización fue contratada al Instituto Forestal por el Proyecto CONAF/PNUD/FAO/CHI/8 - 017, que autoriza su publicación. 


\section{REFERENCIAS BIBLIOGRAFICAS}

1. BASKERVILLE, G. L. 1972. Use of logarithmic regression in the estimation of plant biomass. Can J. For. Res. 2: $49 \cdot 53$.

2. CROW, T. R. AND P.R. LAIDLY. 1980. Alternative models for estimating woody plant biomass. Can. J. For. Res. 10: 367 - 370 .

3. ETIENNE, M.: E. CAVIEDES; C. GONZALEZ Y C. PRADO. 1982, Canografía de la vegetación de la zona árida mediterránea de Chile. Transecto I: Puerto Oscuro. Combarbalá, Monte Patria, Ovalle. La Serena. Terra Aridae I (1): 1 - 73.

4. ETIENNE, M.C. GONZALEZ Y C. PRADO 1982a. Cartografía de la vegetación de la zona árida mediterránea de Chile. Transecto II: Los Vilos. Illapel, Combarbalá, Terra Aridae 1 (2): 81 - 126.

5. IREN- Instituto de Investigaciones de Recursos Naturales. 1979. Estudio de las comunidades Agricolas IV Región. Vegetación. CORFO. 96. p.

6. KOZAK, A. 1970. Methods for ensuring additivity of biomass components by regression analysis. For. Chron. 46(5): $402-404$.

7. RIGGAN, P.J. AND P.H. DUNN. 1982. Harvesting Chaparral biomass for energy. An environmental assessment. Gen. Tech. Rep. PSW - 58. Berkely, CA: Pacific Southwest and Range Experiment Station, Forest Service, U.S.D.A. p. $149 \cdot 157$

8. WIANT, H.V. AND E.J. HARNER. 1979. Percent bias and Standard Error in Logarithmic Regression. For. Sci. 25(1): $167 \cdot 168$.

9. WILLIAMS JR., J.G. 1982. Modeling problems in predicting total-tree and tree - component biomass. In: Proc. of the 1982 Southern Forest Biomass Working Group Workshop. Alexandria, Lousiana. p. 111-115.

10. YANDLE, D.O. AND H.V. WIANT, JR. 1981. Estimation of plant biomass based on the allometric equation. Can. J. For. Res. Vol. 11: 833-834. 